\title{
Enhanced Modelling of a 1-D Phased Ultrasonic Array for Intracorporeal Sonoporation
}

\author{
Alexandru C. Moldovan, Zhen Qiu, David Lines, Anthony Gachagan \\ Centre for Ultrasonic Engineering \\ University of Strathclyde \\ Glasgow, United Kingdom \\ alexandru.moldovan@strath.ac.uk
}

\author{
Sandy Cochran \\ Centre for Medical and Industrial Ultrasonics \\ University of Glasgow \\ Glasgow, United Kingdom
}

\begin{abstract}
Precise control of ultrasonic (US) power is required during sonoporation to ensure pressure amplitude in the target tissue is maintained within the bounds of therapeutic efficiency and below the maximum threshold of safe use. Pressure mapping with a scanning tank and needle hydrophone (NH) represents a convenient means to evaluate US beam profiles and peak negative pressures (PNP) achieved by therapeutic arrays in the medium. However, the method is restricted to fluid media only, and lacks the dynamic capabilities required during the medical procedure. Software modelling can address these issues for dynamic determination of the optimum array driving parameters in relation to therapeutic requirements. This paper describes the correlation between measurements obtained with a scanning tank and the simulation results for four different experimental 1-D phased arrays. Two simulation frameworks were developed using OnScale (Onscale, CA, US) and cross-compared, with the main difference between them being the solver used for modelling US propagation in the medium. The first method used a finite element analysis (FEA) approach for the water and the second method, previously described in [1], relied on Kirchhoff time-domain extrapolation. Electrical cabling was first omitted then included in the model and the outputs were compared with the previous data. Results show that both frameworks led to a similar fit in PNP amplitude with the measurements and that the beam shape strongly agrees with the experimental data in both cases. However, the extrapolation-based method was less computationally demanding than FEA and allowed for modelling larger loads within available computing resources i.e. memory.
\end{abstract}

Keywords - therapeutic ultrasound; sonoporation; 1-D linear phased array; 1-3 piezocomposite; modeling; FEA; extrapolation.

\section{Clinical Background AND Motivation}

Sonoporation is a targeted drug delivery modality that relies on the mechanical effects of focused US and microbubble (MB) cavitation to increase the permeability of the treated tissue, raising its susceptibility to drug absorption. Injected MBs act as cavitation nuclei and lower the PNP threshold required to obtain the expected therapeutic effects. Their oscillations in close proximity to cells have been proven to form pores in the cellular membrane, which led to enhanced drug uptake [2], [3]. The most notable US parameters that influence cavitation magnitude and therefore sonoporation effectiveness are: PNP, center frequency of US, pulse length and pulse repetition frequency [4], [5].

Application of focused US intracorporeally via blood vessels has several advantages over extracorporeal approaches,

Mr. Moldovan's PhD Studentship is funded through an Engineering and Physical Sciences Research Council (EPSRC) Doctoral Training Grant (EP/N509760/1) mainly due to the short US propagation distance, including: reduced driving power required due to lower attenuation in the tissue, easier focusing due to lack of obstacles in the path, more uniform load medium, and reduced motion of the target tissue relative to the transducer. However, array self-heating during insonation and therapeutic power levels require careful control in order to avoid vessel rupture or blood coagulation.

1-D phased arrays offer the benefit of beam focusing and steering in the plane of the aperture, thus improving therapeutic localization of the procedure and increasing the insonated area compared to single element transducers. Assessment of the beam profile and pressure amplitudes attained at focus represents an important tool for therapeutic planning. Generally, experimental beam characterization is performed with a NH in a scanning tank with a large volume of water. However, this method cannot be applied in situ during surgery, which means that any change made to the therapeutic protocol cannot be readily evaluated and relies instead on interpolation or extrapolation of previously collected data. One means to mitigate this issue is to use simulations of the US beam during therapy in order to validate any change in parameters. Yet the reliability of this approach is highly dependent on good correlation with the experimental data.

\section{AIM OF STUDY}

The current work focuses on comparing the simulated beam profiles with the data obtained from the scanning tank. Four types of miniature 1-3 piezocomposite, 1-D phased arrays whose manufacture and characterization have been described previously [1] were used as part of this study.

Two simulation frameworks were employed to model beam patterns achieved by the arrays in a water load. Both frameworks used FEA to solve the electromechanical equations for the piezoelectric materials and to simulate the effect of the backing layer, while the load was either modelled with an explicit solver or with Kirchhoff integral time-domain extrapolation [1]. The efficiency of the two frameworks was evaluated in terms of beam profile shape and PNP fit with the measurement data, and in terms of the computational load, especially limitations on model accuracy and execution time.

The influence of the precision in the representation of the driving signal on the simulated beam profile and PNP was then evaluated in relation to the scanning tank data. This was achieved by cross-comparing the outcomes of either using a 
pure sine wave excitation signal to drive the modelled arrays, or importing the voltage waveform measured from the FI Toolbox into the simulation. Additionally, the effect of including the electrical cabling as part of the driving circuit for the modelled transducers was evaluated.

\section{TECHNICAL BACKGROUND}

The four 1-3 piezocomposite arrays differed in the active material selection (PZT-5H and PMN-29\%PT), and in the resonance frequency $(1.5 \mathrm{MHz}$ and $3.0 \mathrm{MHz})$. The lower frequency arrays comprised 24 elements and the higher frequency arrays had 32 elements. All four arrays had a $10 \mathrm{~mm}$ thick light backing layer [1]. The transducers were driven with a DSL FI Toolbox controller based on a National Instruments FlexRIO platform (Austin, TX, USA).

The arrays were connected to the controller through separate mini-coaxial cables (RG174A/U) with a matched impedance of $50 \Omega$ and a length of $1.0 \mathrm{~m}$. The driving voltage was measured with an oscilloscope set to high input impedance, connected in parallel to one channel of the FI Toolbox prior to each beam pattern characterization in the scanning tank. Due to the close proximity of the transducer to the water, the oscilloscope probe was placed at the DSL connector, before the mini-coaxial cables. In order to account for the effect of the cables, the equivalent electrical circuit of the cables was measured with a calibrated RLC meter.

Beam pattern characterization was performed with a 3-DOF linear stage coupled to a $0.2 \mathrm{~mm} \mathrm{NH}$ (Precision Acoustics, Dorchester, United Kingdom) [1]. Table I summarizes the focal distances and steering angles evaluated.

TABLE I. STEERING ANGLES AND FocAl Distances USED FOR BEAM PROFILING

\begin{tabular}{|c|c|c|}
\hline $\begin{array}{c}\text { Resonance } \\
\text { Frequency }\end{array}$ & Focal Distance (mm) & Steering Angles ( ${ }^{\circ}$ ) \\
\hline \multirow{2}{*}{$1.5 \mathrm{MHz}$} & 5.0 & $0,15,30,40$ \\
\cline { 2 - 3 } & 10.0 & 0 \\
\hline \multirow{2}{*}{$3.0 \mathrm{MHz}$} & 5.0 & $0,15,30,40$ \\
\cline { 2 - 3 } & 8.0 & 0 \\
\hline
\end{tabular}

\section{Methodology}

The mesh size of all FEA models in the array length and elevation directions was set to half the kerf width $\left(\mathrm{k}_{\mathrm{w}}\right)$ as this feature was the finest $\left(\mathrm{k}_{\mathrm{w}}<\lambda / 15\right)$. The mesh size in the thickness direction was relaxed to $0.75 \mathrm{k}_{\mathrm{w}}$ to reduce computational load. The $10 \mathrm{~mm}$ backing layer was fully included in the models for the $1.5 \mathrm{MHz}$ transducers but its thickness was reduced to $7.0 \mathrm{~mm}$ for the $3.0 \mathrm{MHz}$ PZT-5H transducer and to $4.0 \mathrm{~mm}$ for the $3.0 \mathrm{MHz}$ PMN-29\%PT array to avoid memory overflow because of the finer meshing for the higher frequency.

The FEA simulation of wave propagation in the water load was performed in 3D. Time histories of a $2 \mathrm{D}$ slice centered in the middle of the array's elevation and extending in the directions of the transducer length and depth into the medium were recorded. The depth was limited to $10 \mathrm{~mm}$ for the
1.5 MHz arrays when focused at $5.0 \mathrm{~mm}$ and to $20 \mathrm{~mm}$ for the $10 \mathrm{~mm}$ focal distance. The medium was limited to $8 \mathrm{~mm}$ depth for the 3.0 MHz PZT-5H array and a load thicker than $2.5 \mathrm{~mm}$ could not be simulated due to memory overflow for the 3.0 MHz PMN-29\%PT array, rendering the model impractical. The limiting factor on the load depth was the number of FEA mesh elements required by the model. The $3.0 \mathrm{MHz}$ PMN$29 \%$ PT array had the piezocomposite with the lowest kerf size $(28.5 \mu \mathrm{m})$, which increased meshing resolution significantly.

The FEA and extrapolation framework presented previously [1] did not require a full 3D model of the water medium and the extrapolation was performed only for the $2 \mathrm{D}$ slice to minimize computational load. This framework relied on Kirchhoff integral time-domain extrapolation, computed at equally spaced points of the 2D slice. However, a minimal water load of $2.5 \mathrm{~mm}$ depth was still included in the 3D FEA model of the array in order to produce the time histories required to perform the extrapolation in the far-field. The extrapolation reference plane was placed at $100 \mu \mathrm{m}$ axial distance from the transducer surface in the medium. The medium depth modelled with FEA was chosen to provide a minimum separation of $2.5 \lambda$ between the top absorbing boundary of the FEA model and the extrapolation reference plane, to avoid interference from the absorbing boundary.

The driving signal for the modeled arrays used in both frameworks was initially a pure 10-cycle sine wave burst, with $\mathrm{V}_{\mathrm{RMS}}$ equal to the RMS value of the signal measured from the FI Toolbox. Subsequently, the true voltage from the array controller was included in the models. The RLC series circuit of the connecting cables was then included in the two frameworks and modelled for both types of driving voltage.

\section{RESULTS}

In order to evaluate the correlation between the results of the simulation frameworks and the measurement data, two aspects were considered: the fit in beam profile expressed in terms of overlaid $-1 \mathrm{~dB},-3 \mathrm{~dB}$ and $-12 \mathrm{~dB}$ contours and the $\mathrm{PNP}_{-1 \mathrm{~dB}}$ ratio. The latter parameter denotes the average PNP value within the $-1 \mathrm{~dB}$ profile of the beam. This value was chosen instead of the absolute minimum PNP recorded throughout the beam because the $-1 \mathrm{~dB}$ profile denoted a region as opposed to a single point and was considered more relevant to calculation of therapeutic dosage.

For both simulation frameworks, and for all cases of driving signal and cabling, the beam shape comparison with the measured data from the scanning tank was satisfactory. As Fig. 1 shows, the simulations resolved the steering angle and beam contours well. The $-1 \mathrm{~dB}$ and $-3 \mathrm{~dB}$ beam profiles, which are of highest therapeutic relevance, were very similar to the experimental data, and even the $-12 \mathrm{~dB}$ profile showed satisfactory resemblance with the measured dataset. The noise appearing in the $-12 \mathrm{~dB}$ profile produced by the FEA framework was generated by divergence of the solutions to the constitutive equations, which was caused by the rectangular mesh size, with the thickness dimension being 1.5 times coarser than the other two dimensions. 


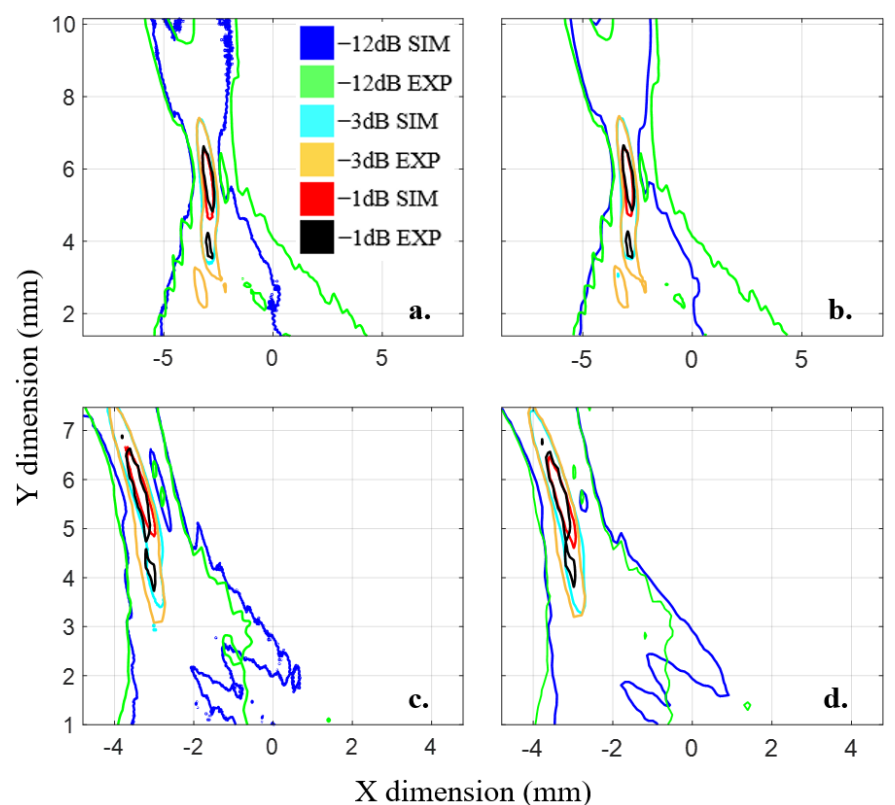

Fig. 1. Beam Profile Comparison between the Simulated (SIM) and the Experimental (EXP) Datasets for: the $1.5 \mathrm{MHz}, P M N-29 \%$ PT Array Steered at $30^{\circ}$ (a. FEA only Framework, b. FEA + Extrapolation), and for the 3.0 MHz, PZT-5H Array (c. FEA only Framework, d. FEA + Extrapolation)

The correlation in pressure amplitudes was computed as the percentage ratio $(R)$ between the $\mathrm{PNP}_{-1 \mathrm{~dB}}$ obtained from measurement with the scanning tank and the simulated $\mathrm{PNP}_{\text {-1dB }}$. The average percentage ratio $(\bar{R})$ over all steering angles and focal distances tested for each simulation profile, and the corresponding standard deviation $(\sigma)$ were calculated with Eq. (1):

$$
\bar{R}=\frac{\sum_{1}^{N} R_{i}}{N}, \quad \sigma=\sqrt{\frac{\sum_{1}^{N}\left(R_{i}-\bar{R}\right)^{2}}{N}}
$$

where $\mathrm{R}=$ the $\%$ ratio of $\mathrm{PNP}_{-1 \mathrm{~dB}}$ between each measurement and simulation, and $\mathrm{N}=$ the number of steering cases evaluated per simulation profile

A summary of the correlation results for all evaluated simulation profiles is provided in Table II. The average difference in $\bar{R}$ between the FEA and the FEA + extrapolation frameworks is $2.33 \%$ and in the average standard deviation is only $0.59 \%$. This result proves that using a combination of FEA for simulating the array and Kirchhoff time-extrapolation for the load is similarly efficient in predicting the beam shape and PNP values at focus with a full FEA approach, but at a lower computational cost, and with potentially higher resolution.

Using an ideal sine wave burst to drive the modelled transducers produced a better fit, in terms of $\mathrm{PNP}_{-1 \mathrm{~dB}}$ with the measured beams, than importing the signal from the FI Toolbox and there was no significant difference in the beam shapes produced by the two simulation profiles.
TABLE II. AVERAge PERCENTAge RATIO AND StANDARD DEVIATION BETWEEN THE EXPERIMENTAL AND THE MODELLED PNP ${ }_{-1 \mathrm{DB}}$

\begin{tabular}{|c|c|c|c|c|c|c|}
\hline \multirow{2}{*}{ Array } & \multirow{2}{*}{$\begin{array}{c}\text { Frame } \\
\text { work }\end{array}$} & & \multicolumn{2}{|c|}{ Sine Burst } & \multicolumn{2}{|c|}{ DSL Waveform } \\
\hline & & & $\begin{array}{c}\text { No } \\
\text { Cables }\end{array}$ & $R L C$ & $\begin{array}{c}\text { No } \\
\text { Cables }\end{array}$ & $R L C$ \\
\hline \multirow{4}{*}{$\begin{array}{l}\text { PZT-5H, } \\
1.5 \mathrm{MHz}\end{array}$} & \multirow{2}{*}{$\begin{array}{l}\text { FEA + } \\
\text { Extrap }\end{array}$} & $\bar{R}$ & 67.5 & 66.7 & 60.9 & 60.2 \\
\hline & & $\sigma$ & 1.96 & 1.93 & 2.15 & 2.15 \\
\hline & \multirow{2}{*}{$\begin{array}{l}\text { FEA } \\
\text { only }\end{array}$} & $\bar{R}$ & 64.8 & 64.0 & 58.5 & 57.7 \\
\hline & & $\sigma$ & 2.62 & 2.59 & 2.38 & 2.35 \\
\hline \multirow{4}{*}{$\begin{array}{c}\text { PMN-PT, } \\
1.5 \mathrm{MHz}\end{array}$} & \multirow{2}{*}{$\begin{array}{l}\text { FEA + } \\
\text { Extrap }\end{array}$} & $\bar{R}$ & 67.9 & 67.5 & 57.8 & 57.5 \\
\hline & & $\sigma$ & 4.23 & 4.19 & 3.29 & 3.19 \\
\hline & \multirow{2}{*}{$\begin{array}{l}\text { FEA } \\
\text { only }\end{array}$} & $\bar{R}$ & 64.8 & 64.5 & 55.9 & 55.6 \\
\hline & & $\sigma$ & 2.88 & 2.86 & 2.31 & 2.38 \\
\hline \multirow{4}{*}{$\begin{array}{l}\text { PZT-5H, } \\
3.0 \mathrm{MHz}\end{array}$} & \multirow{2}{*}{$\begin{array}{l}\text { FEA + } \\
\text { Extrap }\end{array}$} & $\bar{R}$ & 44.9 & 43.0 & 37.2 & 35.5 \\
\hline & & $\sigma$ & 4.28 & 4.20 & 3.46 & 3.44 \\
\hline & \multirow{2}{*}{$\begin{array}{l}\text { FEA } \\
\text { only }\end{array}$} & $\bar{R}$ & 43.0 & 41.0 & 35.2 & 33.6 \\
\hline & & $\sigma$ & 6.90 & 6.67 & 5.86 & 5.74 \\
\hline \multirow{2}{*}{$\begin{array}{l}\text { PMN-PT, } \\
3.0 \mathrm{MHz}\end{array}$} & \multirow{2}{*}{$\begin{array}{l}\text { FEA + } \\
\text { Extrap }\end{array}$} & $\bar{R}$ & \multirow{2}{*}{\multicolumn{2}{|c|}{$\mathrm{N} / \mathrm{A}^{\mathrm{a}}$}} & 80.7 & 74.1 \\
\hline & & $\sigma$ & & & 9.97 & 8.18 \\
\hline
\end{tabular}

a. The 3.0 MHZ PMN-29\%PT array was driven with a lower duty cycle waveform from the FI Toolbox to avoid NH damage, and thus the sine wave equivalence was not relevant, and was not included in the comparison.

The inclusion of the cabling in the model led to a small increase in the simulated PNP values and thus a decrease in $\bar{R}$ in all evaluated profiles. This was generated by the amplification of the FEA modelled driving voltage amplitudes by the oscillatory RLC circuit (Fig 2.). The effect was further increased when a noisier signal was imported into the simulation for the 3.0 MHz PMN-29\%PT array (Fig 2. c-d), which required a lower duty cycle from the FI Toolbox pulsers to avoid NH damage during beam pattern scanning.

\section{DISCUSSION}

Across the entire data set, the average ratio in $\mathrm{PNP}_{-1 \mathrm{~dB}}$ between measurements and simulation was $56.2 \%$ and the average standard deviation was $3.93 \%$. Even if the simulations predicted larger PNPs than the real measurements in all evaluated cases, the low standard deviations made possible the use of a correction factor equal to $\bar{R}$, which could be multiplied by the modelled data to improve the fit in PNP.

Error sources that were attributed to the decrease in the fit between simulation and measurements included: a lower coupling coefficient of the real arrays compared to the modelled ones due to slight mismatch in piezoelectric constants, degradation of active material properties during manufacturing, and effects of bonding interconnects and transducer casing not simulated; a $14 \%$ uncertainty in the $\mathrm{NH}$ sensitivity calibration data; possible water ingress during prolonged scanning periods and other random measurement errors. 

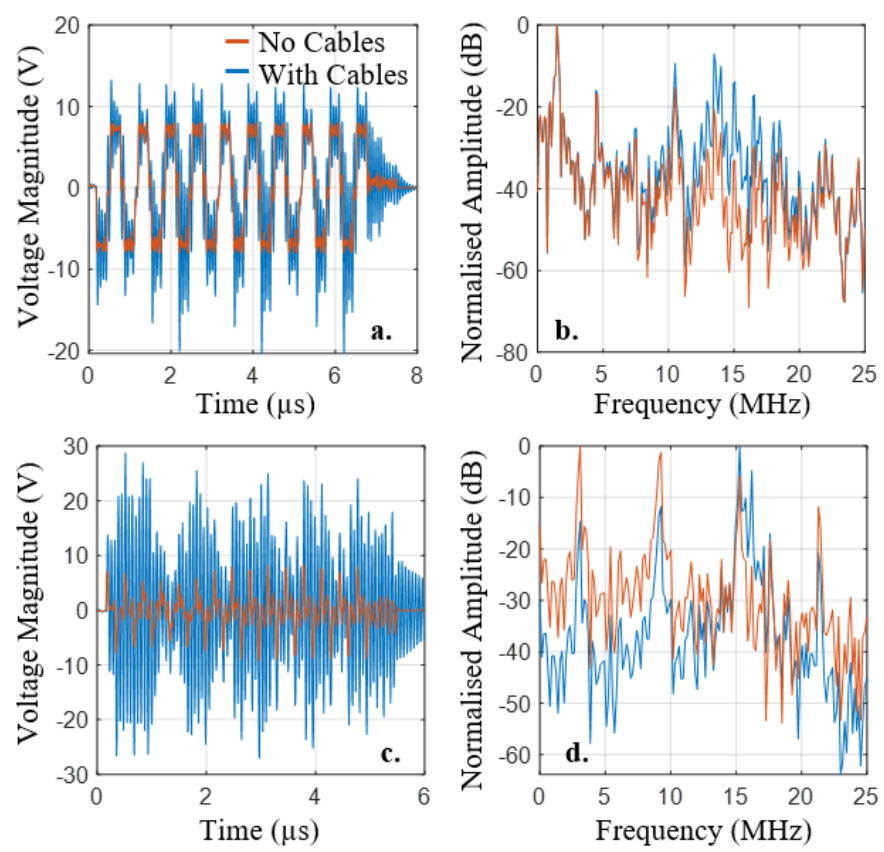

Fig. 2. Effect of Simulating the Array Cables on the Driving Voltage Waveform and its Normalised Spectral Components for: $a .-b$. Normal $1.5 \mathrm{MHz}$ Driving Signal Imported from the FI Toolbox, c.-d. Lower Duty Cycle Signal Used for the 3.0 MHz, PMN-29\%PT Array

Regarding simulation time, the FEA framework took an average of 105 minutes to simulate the $1.5 \mathrm{MHz}$ arrays and 240 minutes for the $3.0 \mathrm{MHz}$ ones. The FEA combined with Kirchhoff extrapolation framework required an average of 60 minutes in total for the $1.5 \mathrm{MHz}$ transducers and 180 minutes for the $3.0 \mathrm{MHz}$ ones on a $32 \mathrm{~GB}$ RAM, 16-core CPU workstation. Furthermore, the FEA only framework was much more demanding in terms of memory because it required a higher number of mesh elements, whereas the FEA + extrapolation framework divided the computation into two separate processes, allowing for increased model resolution or lower execution time.

\section{SUMMARY AND CONCLUSIONS}

This research showed that FEA analysis of US wave propagation in uniform media has similar accuracy to Kirchhoff integral time-extrapolation. The latter method is preferred in terms of lower execution time and lower resource usage. Simulating the entire 3D volume of the load medium with FEA required a large amount of memory, limiting the effective load size and meshing accuracy. As depicted in this work, the PMN-29\%PT 3.0 MHz array could not be simulated with FEA only when coupled to the same medium dimensions as the other configurations because the model size was larger than the $32 \mathrm{~GB}$ of RAM available on the workstation.

The extrapolation approach still relied on FEA to solve the electromechanical equations of the arrays and to simulate their backing materials, but, as a large portion of the medium was not included in the 3D FEA model, the computational load was reduced. Furthermore, once the reduced-size model was run, the time histories produced for the extrapolation reference plane could be used to extrapolate any size or plane in the medium, without the requirement to rerun the FEA analysis, as in the case of the full-FEA framework. The downside of simulating the medium with time extrapolation is that the method does not allow inclusion of features in the medium and it must be uniform (e.g. not containing stacked layers).

Using the excitation signal measured from the array controller in the model led to an increase in the simulated PNP amplitude, thus reducing the fit with the measured data even more than in the case of the sine wave. The addition of the connecting cables as an equivalent RLC circuit in the model also decreased the $\mathrm{PNP}_{-1 \mathrm{~dB}}$ fit, because of an increase in the simulated driving voltage. The beam shapes at each steering angle and focal distance were similar between all simulation profiles and corresponded well with the experimental data. Even if the modeled PNP-1dB was on average almost double the measured value, the low standard deviation between the simulation and measurement data indicates that a correction factor could simply be multiplied by the simulated PNP values to align them with the experimental ones.

Future work will focus on optimizing the piezoelectric material constants of the active phase used for modelling the 1-3 piezocomposite arrays to improve the fit in the impedance curves between simulation and measurement, followed by a similar correlation between the beams produced with the optimized simulation profile and the scanning data.

\section{ACKNOWLEDGMENT}

We are grateful for the OnScale research license provided by OnScale, CA, USA.

\section{REFERENCES}

[1] A. C. Moldovan, Z. Qiu, D. Lines, A. Gachagan, and S. Cochran, "Development of a 1-D Linear Phased Ultrasonic Array for Intravascular Sonoporation," in 2019 IEEE International Ultrasonics Symposium (IUS), Oct. 2019, pp. 2056-2059, doi: 10.1109/ULTSYM.2019.8925549.

[2] K. Kooiman, H. J. Vos, M. Versluis, and N. de Jong, "Acoustic behavior of microbubbles and implications for drug delivery," Adv. Drug Deliv. Rev., vol. 72, pp. 28-48, Jun. 2014, doi: 10.1016/j.addr.2014.03.003.

[3] I. Lentacker, I. De Cock, R. Deckers, S. C. De Smedt, and C. T. W. Moonen, "Understanding ultrasound induced sonoporation: Definitions and underlying mechanisms," Adv. Drug Deliv. Rev., vol. 72, pp. 4964, Jun. 2014, doi: 10.1016/j.addr.2013.11.008.

[4] F. E. Shamout et al., "Enhancement of Non-Invasive Trans-Membrane Drug Delivery Using Ultrasound and Microbubbles During Physiologically Relevant Flow," Ultrasound Med. Biol., vol. 41, no. 9, Art. no. 9, Sep. 2015, doi: 10.1016/j.ultrasmedbio.2015.05.003.

[5] H.-D. Liang, J. Tang, and M. Halliwell, "Sonoporation, drug delivery, and gene therapy," Proc. Inst. Mech. Eng. [H], vol. 224, no. 2, Art. no. 2, Feb. 2010, doi: 10.1243/09544119JEIM565.M. Young, The Technical Writer's Handbook. Mill Valley, CA: University Science, 1989. 